\title{
Studi epidemiologici osservazionali europei: le coorti dalla nascita
}

\author{
Eleonora Morabito*, Claudia Pansieri*, Chiara Pandolfini*, Antonio Clavenna*, \\ Imti Choonara**, Maurizio Bonati* \\ *Laboratorio per la Salute Materno Infantile, Dipartimento di Salute Pubblica, Istituto di Ricerche Farmacologiche \\ Mario Negri IRCCS, Milano; \\ **Academic Division of Child Health, University of Nottingham School of Medicine, Derby DE22 3DT, United Kingdom
}

\begin{abstract}
Obiettivi: negli ultimi anni c'è stato un crescente interesse per le coorti dalla nascita. L'obiettivo di questa revisione della letteratura è di conoscere e comprendere lo stato dell'arte delle coorti europee, con un focus su quelle che hanno iniziato la raccolta dei dati alla nascita. In particolare, lo scopo è quello di fornire un panorama degli attuali argomenti di ricerca e progettazione e fornire input a coloro che creano collaborazioni e pongono linee guida volte a unificare le metodologie per consentire la condivisione dei dati e massimizzare l'acquisizione di conoscenze.
\end{abstract}

Metodi: abbiamo cercato in PubMed ed Embase articoli riferiti alle coorti dalla nascita longitudinali europee e abbiamo cercato anche siti online pertinenti.

Risultati: sono state individuate 111 coorti, 45 delle quali dalla nascita. Queste coorti sono iniziate tra il 1921 e il 2015 e hanno rappresentato 19 Paesi, con diverse dimensioni del campione (da 236 a 21.000 bambini). A1 5 gennaio 2020 stavano ancora reclutando. Le principali aree affrontate sono state le malattie allergiche (14 coorti) e l'esposizione ambientale (coorti 12). La maggior parte delle coorti sono state finanziate con fondi pubblici.

Conclusioni: l'importanza di lunghi periodi di follow-up per identificare fattori di rischio per i disturbi che hanno un'eziologia perinatale/precoci, fanno delle coorti uno strumento per definire il ruolo di fattore genetici psicologici, sociali e ambientali sullo sviluppo. Inoltre, sono necessari metodologie appropriate e comuni per consentire il confronto e la condivisione dei dati delle coorti.

\footnotetext{
Objective: in recent years there has been a growing interest in birth cohorts. The aim of this review is to know and understand the state of the art of European cohorts to date, with a focus on those that started data collection at birth. In particular, the aim is to provide an overview of current research topics and designs, and provide input for those creating collaborations and laying out guidelines aimed at unifying cohort methodologies to enable data merging and maximize knowledge acquisition.

Methods: we searched PubMed and Embase for articles referring to longitudinal, prospective European birth cohorts, and searched online cohort inventories.

Results: we found references to 111 birth cohorts, 45 of which began enrolment at birth. These cohorts began between 1921 and 2015 and represented 19 countries, with varying sample sizes (236 to 21,000 children). As of 5 January 2020, 5 were still recruiting. The main areas addressed were allergic diseases (14 cohorts) and environmental exposure (12 cohorts) and most cohorts were publicly funded.

Conclusion: given the large costs of running cohorts and the importance of long follow-up periods in identifying the risk factors for disorders thought to have a perinatallearly life etiology, current cohorts must be designed to answer research questions considering several aspects, from genetic ones to psychological, social, and environmental ones. Furthermore, universally recognized methodological aspects are needed to permit the comparison and merging of cohort data.
}

\section{Introduzione}

\section{Studio di coorte}

Lo studio di coorte rientra nella categoria degli studi osservazionali e, per definizione, "segue nel tempo un gruppo di persone con caratteristiche definite, con lo scopo di determinare 1'incidenza o la mortalità associate ad alcune malattie specifiche,
Questi studi hanno il vantaggio di poter essere progettati prima e, quindi, di raccogliere dati di esposizione specifici ai fini dello studio, e possono essere più completi. Lo svantaggio però è il lungo periodo di follow-up necessario prima che si verifichino eventi o malattie, il che determina un elevato costo e il rischio di un alto tasso di perdita di partecipazione al follow-up. Non sono pertanto l'ideale per indagare malattie con lunghi periodi di latenza.

Al contrario, negli studi di coorte retrospettivi, si identificano le persone esposte e non esposte sulla base di dati già disponibili, e il tempo di osservazione è parzialmente o completamente rivolto al passato. Quindi, il disegno di questo studio è meno costoso e il tempo di realizzazione è più breve rispetto agli studi prospettici di coorte. Tuttavia, lo svantaggio principale è il controllo limitato che lo sperimentatore ha sulla raccolta dei dati: importanti fattori confondenti potrebbero non essere stati valutati, oppure i dati esistenti possono essere incompleti, imprecisi o misurati in modo incoerente tra i soggetti.

Il termine retrospettivo non deve far confondere con lo studio caso-controllo che si distingue dallo studio di coorte retrospettivo per la presenza di un gruppo caso e un gruppo controllo, suddivisi sulla base dell'outcome, e non dell'esposizione. Per questo motivo sarebbe più corretto chiamare gli studi retrospettivi di coorte con il termine "studi di coorte storici" [4].

\section{Studio di coorte dalla nascita}

Gli studi di coorte dalla nascita sono degli studi di coorte che seguono l'individuo a partire dal parto o dalla gravidanza, per un lungo periodo di tempo, spesso fino all'adolescenza o all'età adulta. Proprio perché i soggetti sono seguiti longitudinalmente per tutta la loro vita sin dalla nascita, queste coorti consentono di delineare le associazioni tra le prime esposizioni e gli esiti successivi. Sono, quindi, il tipo di disegno più appropriato per determinare la relazione causale tra potenziali fattori di rischio durante il periodo prenatale o postnatale e 
lo stato di salute del neonato, fino all'infanzia e potenzialmente all'età adulta [5].

\section{Un crescente interesse}

Il crescente interesse per gli studi di coorte è dato dal fatto che è ormai noto che le esposizioni nel periodo prenatale e nei primi anni di vita, come i fattori ambientali e familiari, possano influenzare la crescita e lo sviluppo nella vita fetale e nell'infanzia, e la salute durante tutto il corso della vita [6]. Le coorti dalla nascita sono fondamentali per comprenderne gli effetti, nonché i potenziali interventi correttivi. Le prove scientifiche, infatti, hanno dimostrato come semplici azioni che comportano la riduzione dell'esposizione a fattori di rischio o la promozione di fattori protettivi nei primi anni di vita possano prevenire significativamente problemi di salute [7-9].

In questo contesto di crescente attività di ricerca osservata a livello globale l'Europa, e in particolare l'Europa settentrionale, è stata particolarmente attiva.

\section{Obiettivi}

Le coorti possono differire tra loro nella metodologia e nel disegno di studio, in quanto non esistono linee guida comunemente riconosciute per la raccolta dei dati. E ormai esperienza comune che le singole coorti permettono di apportare importanti contributi alla comprensione delle cause ambientali delle malattie infantili e di alcune malattie tuttavia, il loro pieno potenziale può essere realizzato solo con approcci condivisi tra i vari studi [10]. A questo proposito, nella presente revisione sono stati analizzati i disegni di studio delle coorti europee il cui obiettivo è generare un panorama degli attuali argomenti di ricerca e progettazione delle coorti di nascita e fornire input per coloro che creano collaborazioni e definiscono linee guida. Queste ultime sono volte a unificare le metodologie di coorte, per consentire la condivisione dei dati e massimizzare l'acquisizione di conoscenze. Abbiamo inoltre valutato quante coorti dalla nascita affrontano l'impatto del contesto familiare nurturing care e il contributo dell'assistenza dei pediatri sulla salute e la crescita dei bambini, al fine di fornire indicazioni per futuri studi di coorte.

\section{Materiali e metodi}

Tra gennaio e luglio 2019, abbiamo eseguito una revisione delle coorti dalla nascita europee prendendo in considerazione più fonti. Abbiamo cercato su PubMed ed Embase con l'ultimo aggiornamento il $1^{\circ}$ luglio 2019, limitando i risultati al 20 maggio 2019, senza restrizioni sugli an- ni di pubblicazione precedenti. Al fine di non escludere pubblicazioni pertinenti, abbiamo anche consultato siti di coorte dalla nascita online. In particolare, è stata consultata la banca dati web-based (http:// www.birthcohorts.net), creata nell'ambito della rete Children Geno (http://www. chicosproject.eu). Abbiamo anche cercato le coorti elencate da due progetti di ricerca finanziati dall'UE: il progetto ENRIECO [11] e la rete EUCCONET [12].

I criteri di inclusione erano: coorti dalla nascita che avevano sede in un Paese europeo e raccoglievano dati longitudinali e prospettici. I criteri di esclusione: studi sui vaccini, studi caso controllo progettati all'interno di coorti esistenti, studi che applicavano l'analisi genica o altri criteri nella selezione dei campioni, o studi di coorte incentrati solo sui genitori o sugli esiti della gravidanza, che erano esclusivamente retrospettivi, che raccoglievano dati dai registri o che non prevedevano un follow-up.

Abbiamo eseguito analisi più dettagliate sul sottogruppo di coorti che ha iniziato il reclutamento alla nascita e non durante la gravidanza. Sono state incluse le coorti che hanno iniziato a raccogliere dati dopo alcuni mesi di nascita, anche se i pazienti sono stati arruolati alla nascita. Per le analisi più dettagliate è stato spesso necessario cercare ulteriori pubblicazioni scientifiche risultanti dalle singole coorti, oltre ai siti web delle coorti, al fine di limitare dati mancanti.

Di ciascuna coorte sono stati valutati le nazioni coinvolte, l'anno di inizio, la durata del follow-up, la dimensione campionaria, l'attuale stato, il metodo di raccolta dei dati, gli obiettivi e le aree considerate. Il tipo di finanziamento ricevuto dalle coorti è stato classificato in quattro tipi: pubblico (ministeri della sanità, ospedali, compresi gli ospedali universitari, ecc.), fondazione, università e industria.

\section{Risultati}

Sono stati identificati un totale di $111 \mathrm{co}^{-}$ orti dalla nascita europee. Di queste, 66 hanno iniziato l'arruolamento in gravidanza e 45 alla nascita o subito dopo.

\section{Il panorama europeo}

Le 111 coorti europee rappresentavano 27 Paesi, incluse tre nazioni presenti solo nelle quattro coorti multinazionali (Austria, Islanda e Slovenia). I Paesi più frequentemente coinvolti, in 16 coorti ciascuno, sono stati la Germania e il Regno Unito, seguiti dai Paesi Bassi. Il numero di bambini reclutati nelle diverse coorti variava da 107 a 108.500 (mediana 1.924). L'anno di inizio dell'arruolamento nelle diverse coorti variava dal 1921 al 2016 (mediana 2002) e la durata dell'arruolamento, escludendo 10 con iscrizione attualmente in corso, e uno con dati mancanti, variava da 1 a 23 anni (mediana 2). Per quanto riguarda $\mathrm{i}$ follow-up, 62 hanno follow-up in corso, di cui 22 sono permanenti e il resto ha una durata da 1 a 31 anni.

Le 45 coorti che hanno iniziato il reclutamento alla nascita

Le coorti che hanno iniziato il reclutamento alla nascita sono 45, che rappresentano 19 Paesi europei.

Solo la coorte Europrevall è multinazionale e coinvolgeva 9 Paesi. Più di un terzo delle coorti è stato stabilito nell'Europa settentrionale, dove questo tipo di studio ha una lunga tradizione.

La dimensione del campione di ciascuna coorte variava notevolmente, da 236 della coorte olandese del 1990 a oltre 21.000 bambini dello studio TEDS-Twins, con una media di 4230 (mediana 2.515). Le due più grandi coorti di nascita si trovano nel Regno Unito (studio TEDS-Twins con 21.000 bambini iscritti) e in Francia (ELFE, Étude Longitudinale Française depuis l'Enfance, con 18.326 bambini iscritti). Gli anni di inizio delle coorti andavano dal 1921 al 2015 (mediana 2002). Oltre la metà delle coorti è iniziata nel 2000 o successivamente. A partire da gennaio 2020, la maggior parte delle coorti ha chiuso il reclutamento. Cinque coorti stanno ancora reclutando: DONALD (iniziato nel 1985), GUS, Growing Up in Scotland (2004), KUNO-Kids (2015), the LucKi birth cohort (2006) e MUBICOS (2009).

Per quanto riguarda il follow-up, il 49\% delle coorti è ancora in fase di follow-up, mentre il resto è definitivamente chiuso. Tra le coorti più recenti, sono attualmente in corso 15 delle 26 (58\%) coorti costituite dal 2000 in poi e 6 delle 8 (75\%) dal 2010 in poi.

La durata minima dei follow-up era di un anno; per alcuni studi il periodo dichiarato era tutta la vita.

Il fatto che più della metà delle coorti abbia avuto inizio dal 2000 e che il follow-up di molte sia ancora in corso suggerisce che esiste un interesse attivo e attuale per le coorti dalla nascita, sebbene con il coinvolgimento di soli 9 Paesi, e con obiettivi differenti. Gli obiettivi alla base della creazione delle coorti, infatti, sono vari e coprono un'ampia gamma di aspetti della salute dei bambini. La maggior parte delle coorti si è occupata di più aree con la finalità di testare un'ampia gamma di ipotesi. Nel tempo, le priorità di ricerca sono cambiate e negli ultimi anni le aree più comu- 
TABELLA 1. Le 45 coorti europee

\begin{tabular}{|l|l|l|l|}
\hline Nazione & N. di coorti & $\begin{array}{l}\text { Popolazione } \\
\text { della coorte }\end{array}$ & $\begin{array}{l}\text { Anni di inizio } \\
\text { delle coorti }\end{array}$ \\
\hline Belgio & 2 & $1.080 ; 1.196$ & $2002 ; 2010$ \\
\hline Repubblica Ceca & 1 & 7.577 & 1994 \\
\hline Danimarca & 2 & $562 ; 6.090$ & $1998 ; 2000$ \\
\hline Finlandia & 2 & $5.356 ; 9.479$ & $1981 ; 1985$ \\
\hline Francia & 5 & $302-18.326$ & $2003-2012$ \\
\hline Germania & 7 & $1.006-5.991$ & $1990-2015$ \\
\hline Italia & 4 & $697-3.328$ & $2003-2011$ \\
\hline Multinazionale & 1 & 12.049 & 2005 \\
\hline Norvegia & 2 & $2.000 ; 3.754$ & $1992 ; 2003$ \\
\hline Portogallo & 1 & 8.647 & 2005 \\
\hline Slovacchia & 2 & $1.134 ; 1.990$ & $1997 ; 2002$ \\
\hline Svezia & 3 & $2.026-16.058$ & $1994-2007$ \\
\hline Paesi Bassi & 4 & $236-5.000$ & $1977-2006$ \\
\hline Turchia & 1 & 1.377 & 2010 \\
\hline Regno Unito & 8 & $308-21.000$ & $1921-2007$ \\
\hline
\end{tabular}

nemente affrontate sono le malattie allergiche e l'esposizione ambientale. Questo riflette una crescente attenzione agli effetti negativi dell'inquinamento sulla salute. La crescita è stata studiata maggiormente dalle coorti più anziane, mentre l'obesità è una nuova area di ricerca, sebbene tutte le aree rimangano attualmente argomenti di interesse per la ricerca, per l'espansione delle conoscenze e per l'adeguatezza degli interventi. Invece, poche coorti hanno seguito in dettaglio lo sviluppo del bambino e il suo neurosviluppo. Recentemente, si sono aggiunti nell'interesse scientifico nuovi fattori di stress, che si sommano a quelli già identificati e che erano già stati riconosciuti come possibili cause di malattie ed eventi avversi [12]: le circostanze socioeconomiche, la migrazione, l'ambiente urbano e i determinanti legati allo stile di vita [13]. I risultati della revisione mostrano che le misure epigenetiche e fenotipiche e le loro associazioni con gli esiti sulla salute, sin dal concepimento e/o dalla nascita, sono obiettivi crescenti degli studi prospettici di coorte. La raccolta di campioni biologici, è diventata sempre più parte delle coorti [14]. I campioni biologici, infatti, consentono di studiare come i fattori sociali e ambientali lascino impronte biologiche, indipendenti o in associazione con il background genetico [15]. I campioni biologici più frequentemente raccolti sono sangue materno, sangue del cordone ombelicale e urine.

Le coorti che hanno indagato almeno in parte il contesto familiare nurturing care sono poche e relativamente recenti. Sono so- lo tre: ELFE (Étude Longitudinale Française depuis l'Enfance) del 2011, Epifane del 2012 e GUS (Growing up in Scotland) del 2004. Infatti solo negli ultimi anni si sta comprendendo che il contesto familiare è importante [16] e dovrebbe essere un'area di studio prioritaria, considerando anche le disuguaglianze presenti tra le famiglie e l'influenza che i diversi contesti possono avere sull'intera vita sin dalla nascita [17].

\section{Metodologia}

Le coorti sono state sostenute per metodologia e organizzazione da fondi pubblici limitati, e questo ha inevitabilmente influenzato il tipo di coorte. La maggior parte delle coorti ha raccolto dati utilizzando questionari predefiniti e interviste, mentre in poco più della metà delle coorti sono state svolte valutazioni cliniche. Inoltre i Paesi nordici spesso hanno tratto i dati dei pazienti da diversi registri facilitando la raccolta di informazioni anche cliniche e riducendo i costi [18-19]. Un'altra modalità, che è stata applicata da molte coorti, è l'utilizzo delle cartelle cliniche per ottenere dati pertinenti le madri, la gravidanza e il parto. Anche l'uso di questionari basati sul web nella valutazione dei risultati perinatale è risultato un modo valido per raccogliere dati, limitando al contempo i costi [20]. Poche coorti, invece, hanno attivamente coinvolto come persona responsabile della raccolta dati il pediatra di famiglia o il medico di base, a seconda del sistema di assistenza primaria pediatrica nazionale evidenziando il fatto che l'assistenza primaria è una risorsa trascurata per la ricer- ca [21]. Sebbene, l'assistenza primaria preveda un'assistenza completa, coordinata, preventiva e pianificata nel contesto clinico, sociale e scolastico [22]. Il pediatra di famiglia è garante del benessere del bambino e figura di supporto per i genitori. In quanto tale, svolge un ruolo fondamentale nel descrivere l'importanza per le famiglie di azioni specifiche da intraprendere per garantire la salute lo sviluppo del bambino [23]. In tale contesto, la partecipazione dei pediatri di famiglia agli studi di coorte dalla nascita dovrebbe essere considerata come un'iniziativa da implementare.

\section{Conclusioni}

I risultati di questo studio mostrano che le coorti attuali devono essere in grado di rispondere alle prossime domande di ricerca considerando diversi aspetti: condizioni genetiche, biologiche, ambientali, psicologiche/sociali, cure mediche e farmaci, stile di vita.

$\mathrm{Nel}$ prossimo futuro un maggior numero di Paesi dovrebbe essere coinvolto in studi multinazionali, con aspetti metodologici adeguati e universalmente riconosciuti (per esempio: dimensione del campione numerosità e tipo di dati e durata del follow-up), con priorità sanitarie comuni. Il ruolo della Commissione Europea, oltre a sostenere l'attivazione delle coorti multinazionali, è quello di promuovere e massimizzare l'acquisizione di conoscenze volte a migliorare la salute della popolazione.

Riteniamo inoltre che l'assistenza primaria debba essere sostenuta e valorizzata nella ricerca di sanità pubblica. Gli studi futuri dovrebbero comportare una stretta collaborazione con pediatri di famiglia o medici di base. In questa nuova visione il loro ruolo non si limiterà più al trattamento delle malattie, ma comporterà l'assistenza globale del bambino e della famiglia.

Nota. Il presente articolo è parte di:

- Pansieri C, Pandolfini C, Clavenna A, Choonara I, Bonati M. Int J Environ Res Public Health. 2020 Apr 28;17(9):3071.

- La tesi di laurea di Eleonora Morabito Nascere e crescere in Italia oggi: dall'osservatorio ambulatoriale del pediatra di famiglia, Università degli studi di Milano-Bicocca, Dipartimento di Medicina e Chirurgia, Corso di Laurea Magistrale in Medicina e Chirurgia, a.a. 2019-2020.

eleonora.morabito@guest.marionegri.it

La bibligrafia è consultabile online. 
1. Morabia A. A History of Epidemiologic Methods and Concepts. Birkhaeuser Verlag, 2004.

2. Song JW, Chung KC. Observational studies: cohort and case-control studies. Plast Reconstr Surg 2010;126:2234-42.

3. Euser AM, Zoccali C, Jager KJ, Dekker FW. Cohort studies: prospective versus retrospective. Nephron Clin Pract 2009;113:c214-7.

4. Klebanoff MA, Snowden JM. Historical (retrospective) cohort studies and other epidemiologic study designs in perinatal research. Am J Obstet Gynecol 2018;219:447-50.

5. Richmond RC, Al-Amin A, Smith GD, Relton CL. Approaches for drawing causal inferences from epidemiological birth cohorts: a review. Early Hum Dev 2014;90:769-80.

6. Barker DJ. The fetal and infant origins of adult disease. BMJ 1990;301:1111.

7. Balbus JM, Barouki R, Birnbaum LS et al. Early-life prevention of non-communicable diseases. Lancet 2013;381:3-4.

8. Barouki R, Gluckman PD, Grandjean P, Hanson M, Heindel JJ. Developmental origins of noncommunicable disease: implications for research and public health. Environ Health 2012;11:42.

9. World Health Organization Regional Office for Europe Review of Evidence on Health Aspects of Air Pollution-REVIHAAP Project Technical Report.

2013. http://www.euro.who.int/_data/assets/pdf_file/0004/193108/REVIHAAP-Final-technical-report-finalversion.pdf. (Ultimo accesso 24 marzo 2021)

10. Vrijheid M, Casas M, Bergström A et al. European birth cohorts for environmental health research.

Environ Health Perspect 2012;120:29-37.

11. Enrieco. Rischi per la salute ambientale nelle coorti di nascita europee. https://www.enrieco.org (Ultimo accesso 24 marzo 2021)

12. Power C, Elliott J. Cohort profile: 1958 British birth cohort (National Child Development Study). Int J Epidemiol 2006;35:34-41.

13. Lynch J, Smith GD. A life course approach to chronic disease epidemiology. Annu Rev Public Health 2005;26:1-35.

14. Bailey SR, Townsend CL, Dent H, et al. A pilot study to understand feasibility and acceptability of stool and cord blood sample collection for a large-scale longitudinal birth cohort. BMC Pregnancy Childbirth 2017; $17: 439$.

15. Richmond RC, Al-Amin A, Smith GD, Relton CL. Approaches for drawing causal inferences from epidemiological birth cohorts: a review. Early Hum Dev 2014;90:769-80.

16. Britto PR, Lye SJ, Proulx K et al.; Early Childhood Development Interventions Review Group, for the Lancet Early Childhood Development Series Steering Committee. Nurturing care: promoting early childhood development. Lancet. 2017;389:91-102.

17. Black MM, Walker SP, Fernald LCH et al.; Lancet Early Childhood Development Series Steering Committee. Early childhood development coming of age: science through the life course. Lancet. 2017;389:77-90.

18. Doyle A, Golding J. The costing and funding of longitudinal birth cohort studies. Paediatr. Périnat. Epidemiol 2009;23:86-92.

19. Sørensen H.T. Regional administrative health registries as a resource in clinical epidemiology. Int. J. Risk Saf. Med 1997;10:1-22.

20. van Gelder MMHJ, Vorstenbosch S, Derks L, Te Winkel B, van Puijenbroek EP, Roeleveld N. Webbased questionnaires to assess perinatal outcome proved to be valid. J Clin Epidemiol. 2017;90:136-43.

21. Jones N. Primary care is a neglected resource for clinical research. BMJ 2018;361:k1946.

22. Starfield B, Shi L, Macinko J. Contribution of primary care to health systems and health. Milbank Q. 2005;83:457-502.

23. Bhutta ZA, Ahmed T, Black RE, et al.; Maternal and Child Undernutrition Study Group. What works? Interventions for maternal and child undernutrition and survival. Lancet. 2008;371:417-40. 